Bull. Korean Math. Soc. 50 (2013), No. 6, pp. 2013-2020

http://dx.doi.org/10.4134/BKMS.2013.50.6.2013

\title{
SOME PROPERTIES OF GENERALIZED LOCAL HOMOLOGY AND COHOMOLOGY MODULES
}

\author{
Tran Tuan Nam and Nguyen Duc Minh
}

\begin{abstract}
We study some properties of representable generalized local homology modules. By duality, we get some properties of good generalized local cohomology modules.
\end{abstract}

\section{Introduction}

Let $I$ be an ideal of a local Noetherian commutative ring $R$ and $M, N R$ modules. In [8], [10] we defined the $i$-th generalized local homology module $H_{i}^{I}(M, N)$ of $M, N$ with respect to $I$ by

$$
H_{i}^{I}(M, N)={\underset{\leftarrow}{\leftarrow}}_{\lim _{i}} \operatorname{Tor}_{i}^{R}\left(M / I^{t} M, N\right) .
$$

This definition is in some sense dual to J. Herzog's definition of generalized local cohomology modules [5] and in fact a generalization of the usual local homology modules

$$
H_{i}^{I}(M)={\underset{\iota}{t}}_{\lim } \operatorname{Tor}_{i}^{R}\left(R / I^{t} R, M\right)([3],[4]) .
$$

In [7] Macdonald defined a non-zero $R$-module $M$ to be secondary if its multiplication endomorphism by any element $x$ of $R$ is either surjective or nilpotent. It is immediate that the nil-radical of $M$ is a prime ideal $\mathfrak{p}$ and $M$ is call $\mathfrak{p}$-secondary. A secondary representation for an $R$-module $M$ is an expression for $M$ as a finite sum of secondary modules. If such a representation exists, we will say that $\mathrm{M}$ is representable. For the convenient, a zero module is considered as a representable module. If $M$ has a reduced secondary representation $M=M_{1}+M_{2}+\cdots+M_{n}$ and $N_{i}$ is $\mathfrak{p}_{i}$-secondary, we write $\operatorname{Att}(M)=\left\{\mathfrak{p}_{1}, \mathfrak{p}_{2}, \ldots, \mathfrak{p}_{n}\right\}$.

In $[3,4.7]$ we showed that for any artinian $R$-module $M$,

$$
\inf \left\{i: H_{i}^{I}(M) \text { is not } \operatorname{artinian}\right\}=\inf \left\{i: I \nsubseteq \sqrt{\operatorname{Ann}\left(H_{i}^{I}(M)\right)}\right\} .
$$

Received September 10, 2012.

2010 Mathematics Subject Classification. 13D45, 16E30.

Key words and phrases. Artinian module, finitely generated module, representable module, local homology, local cohomology. 
Then Rezaei [12] extended the above result and proved that

$$
\inf \left\{i: H_{i}^{I}(M) \text { is not representable }\right\}=\inf \left\{i: I \nsubseteq \sqrt{\operatorname{Ann}\left(H_{i}^{I}(M)\right)}\right\} .
$$

In this paper, we study representable generalized local homology modules $H_{i}^{I}(M, N)$ and get some general results. The first main result is Theorem 2.4 in which we show that if $M$ is a non-zero representable $I$-separated $R$-module, then

$$
I \subseteq \bigcap_{\mathfrak{p} \in \operatorname{Att}(M)} \mathfrak{p}=\sqrt{\left.\operatorname{Ann}_{R}(M)\right)}
$$

Next, Theorem 2.7 gives us the following equalities when $M$ is finitely generated and $N$ is artinian:

$$
\begin{aligned}
\inf \left\{i: I \nsubseteq \sqrt{\operatorname{Ann}\left(H_{i}^{I}(M, N)\right)}\right\} & =\inf \left\{i: H_{i}^{I}(M, N) \text { is not I-stable }\right\} \\
& =\inf \left\{i: H_{i}^{I}(M, N) \text { is not artinian }\right\} \\
& =\inf \left\{i: H_{i}^{I}(M, N) \text { is not representable }\right\} .
\end{aligned}
$$

In Theorem 2.8 we see that if

$$
t=\sup \left\{i: H_{i}^{I}(M, N) \text { is not representable }\right\} \geq p d(M),
$$

then $H_{t}^{I}(M, N) \neq 0$.

By duality, we get some properties of good generalized local cohomology modules $H_{I}^{i}(M, N)$ (Theorems 3.2 and 3.3). Theorem 3.3 especially gives us a nice consequence: If $M$ and $N$ are finitely generated $R$-modules such that $\operatorname{dim} N=d$ and $\operatorname{ext}^{+}\left(M, H_{I}^{d}(N)\right)=p d(M)=r<\infty$, then $H_{I}^{r+d}(M, N)$ is not good (Corollary 3.4).

\section{Representable generalized local homology modules}

We first recall some basic properties of generalized local homology modules $H_{i}^{I}(M, N)$ that we shall use.

Lemma 2.1 ([8, 2.7]). Let $M$ be a finitely generated $R$-module and $N$ an artinian $R$-module. If $N$ is complete with respect to $I$-adic topology (i.e., $\left.\Lambda_{I}(N) \cong N\right)$, then there is an isomorphism for all $i \geq 0$,

$$
\operatorname{Tor}_{i}^{R}(M, N) \cong H_{i}^{I}(M, N) .
$$

Theorem 2.2 ([8, 2.12]). Let $M$ be a finitely generated $R$-module and $N$ an artinian $R$-module. Let $s$ be a positive integer. Then the following statements are equivalent:

(i) $H_{i}^{I}(M, N)$ is artinian for all $i<s$;

(ii) $I \subseteq \sqrt{\operatorname{Ann}_{R}\left(H_{i}^{I}(M, N)\right)}$ for all $i<s$.

Let $p d(M)$ be the projective dimension of $M$ and $\operatorname{Ndim} N$ the Noetherian dimension of $N$, we have a vanishing theorem for generalized local homology modules. 
Theorem 2.3 ([9, 3.11]). Let $M$ be a finitely generated $R$-module with $p d(M)$ $<\infty$ and $N$ an artinian $R$-module. Then

$$
H_{i}^{I}(M, N)=0
$$

for all $i>p d(M)+\operatorname{Ndim} N$.

Let $I$ be an ideal of $R$, an $R$-module $M$ is called $I$-separated if $\cap_{t>0} I^{t} M=0$. Note that the generalized local homology modules $H_{i}^{I}(M, N)$ are $I$-separated $([8,2.3(\mathrm{i})])$. We have the following properties of non-zero representable $I$ separated $R$-modules.

Theorem 2.4. Let $M$ be a non-zero $I$-separated $R$-module. If $M$ is representable, then

$$
I \subseteq \bigcap_{\mathfrak{p} \in \operatorname{Att}(M)} \mathfrak{p}=\sqrt{\operatorname{Ann}_{R}(M)}
$$

Proof. It follows from $[2,7.2 .11]$ that

$$
\bigcap_{\mathfrak{p} \in \operatorname{Att}(M)} \mathfrak{p}=\sqrt{\operatorname{Ann}_{R}(M)} .
$$

We now assume that

$$
M=T_{1}+T_{2}+\cdots+T_{m}
$$

is a minimal secondary representation of $M$ and $T_{j}$ is $\mathfrak{p}_{j}$-secondary for $j=$ $1,2, \ldots, m$. Then $\operatorname{Att}(M)=\left\{\mathfrak{p}_{1}, \mathfrak{p}_{2}, \ldots, \mathfrak{p}_{m}\right\}$.

If $I \nsubseteq \bigcap_{j=1}^{m} \mathfrak{p}_{j}$, then $I \nsubseteq \mathfrak{p}_{s}$ for some $s \in\{1,2, \ldots, m\}$. Thus there exists $x \in I-\mathfrak{p}_{s}$. It follows

$$
T_{s}=x T_{s}=\bigcap_{t>0} x^{t} T_{s} \subseteq \bigcap_{t>0} x^{t} M=0
$$

which is a contraction. The proof is complete.

By $[8,2.3(\mathrm{i})]$ the generalized local homology module $H_{i}^{I}(M, N)$ is $I$-separated, so we have the following immediate consequence.

Corollary 2.5. Let $M$ and $N$ be $R$-modules and $i$ an integer. If $H_{i}^{I}(M, N)$ is a representable $R$-module, then

$$
I \subseteq \sqrt{\operatorname{Ann}_{R}\left(H_{i}^{I}(M, N)\right)} .
$$

An $R$-module $N$ is called $I$-stable if for each element $x \in I$, there is a positive integer $n$ such that $x^{t} N=x^{n} N$ for all $t \geq n$. There are many $I$-stable modules. For example, for an $R$-module $M$ the quotient module $N=M / I M$ is $I$-stable. Artinian modules especially are $I$-stable (see [10]). To prove Theorem 2.7 we need the following lemma.

Lemma 2.6. Let $M$ be an I-separated $R$-module. Then $M$ is I-stable if and only if $I \subseteq \sqrt{\operatorname{Ann}_{R}(M)}$. 
Proof. "If" is clear.

"Only if". Note that $I$ is finitely generated, as $R$ is Noetherian. Since $M$ is $I$-stable, there is a positive integer $n$ such that

$$
I^{n} M=I^{n+1} M=\bigcap_{t>0} I^{t} M=0 .
$$

Thus $I \subseteq \sqrt{\operatorname{Ann}_{R}(M)}$.

Theorem 2.7. Let $M$ be a finitely generated $R$-module and $N$ an artinian $R$-module. Let $s$ be a positive integer. Then the following statements are equivalent:

(i) $H_{i}^{I}(M, N)$ is I-stable for all $i<s$;

(ii) $H_{i}^{I}(M, N)$ is artinian for all $i<s$;

(iii) $H_{i}^{I}(M, N)$ is representable for all $i<s$.

Proof. (i) $\Rightarrow$ (ii) by Theorem 2.2 and Lemma 2.6.

(ii) $\Rightarrow$ (iii) is clear, as every artinian module is representable.

(iii) $\Rightarrow$ (i) by Corollary 2.5 and Lemma 2.6.

In the following theorem we find some equivalent conditions when generalized local homology modules $H_{i}^{I}(M, N)$ are representable for all $i>p d(M)+s$.

Theorem 2.8. Let $M$ be a finitely generated $R$-module and $N$ an artinian $R$-module. Let $s$ be a non-negative integer. Then the following statements are equivalent:

(i) $H_{i}^{I}(M, N)$ is I-stable for all $i>p d(M)+s$;

(ii) $H_{i}^{I}(M, N)$ is artinian for all $i>p d(M)+s$;

(iii) $\operatorname{Ass}_{R}\left(H_{i}^{I}(M, N)\right) \subseteq\{\mathfrak{m}\}$ for all $i>p d(M)+s$;

(iv) $H_{i}^{I}(M, N)=0$ for all $i>p d(M)+s$;

(v) $H_{i}^{I}(M, N)$ is representable for all $i>p d(M)+s$.

Proof. (i) $\Rightarrow$ (ii). We proceed by induction on $d=\operatorname{Ndim} M$.

If $d=0, H_{i}^{I}(M, N)=0$ for all $i>p d(M)$ by Theorem 2.3 and we have the result.

Let $d>0$. There is a positive integer $n$ such that $I^{t} N=I^{n} N$ for all $t \geq n$. Set $K=I^{n} N$, the short exact sequence of artinian $R$-modules

$$
0 \longrightarrow K \longrightarrow N \longrightarrow N / K \longrightarrow 0
$$

induces an exact sequence of generalized local homology modules

$$
\cdots \rightarrow H_{i+1}^{I}(M, N / K) \rightarrow H_{i}^{I}(M, K) \rightarrow H_{i}^{I}(M, N) \rightarrow H_{i}^{I}(M, N / K) \rightarrow \cdots .
$$

It is clear that $N / K$ is complete in the $I$-adic topology. By Lemma 2.1, there is an isomorphism $H_{i}^{I}(M, N / K) \cong \operatorname{Tor}_{i}^{R}(M, N / K)=0$ for all $i>p d(M)$. Then $H_{i}^{I}(M, K) \cong H_{i}^{I}(M, N)$ for all $i>p d(M)$. Thus, the proof will be complete if we show that $H_{i}^{I}(M, K)$ is artinian for all $i>p d(M)+s$. As $K$ is an artinian $R$-moduls and $I K=K$, there is an element $x \in I$ such that $x K=K$. Moreover, 
there is a positive integer $r$ such that $x^{r} H_{i}^{I}(M, K)=0$ for all $i>p d(M)+s$. Now the short exact sequence

$$
0 \longrightarrow 0: K x^{r} \longrightarrow K \stackrel{\cdot x^{r}}{\longrightarrow} K \longrightarrow 0
$$

induces a short exact sequence of generalized local homology modules

$$
0 \longrightarrow H_{i+1}^{I}(M, K) \longrightarrow H_{i}^{I}\left(M, 0:_{K} x^{r}\right) \longrightarrow H_{i}^{I}(M, K) \longrightarrow 0
$$

for all $i>p d(M)+s$. It follows $I \subseteq \sqrt{\operatorname{Ann}_{R}\left(H_{i}^{I}\left(M, 0:_{K} x^{r}\right)\right)}$ for all $i>$ $p d(M)+s$. That means $H_{i}^{I}\left(M, 0:_{K} \overline{x^{r}}\right)$ is $I$-stable for all $i>p d(M)+s$. By the inductive hypothesis, $H_{i}^{I}\left(M, 0:_{K} x^{r}\right)$ is artinian and then $H_{i}^{I}(M, K)$ is also artinian for all $i>p d(M)+s$.

(ii) $\Rightarrow$ (iii) is clear.

(iii) $\Rightarrow$ (iv). We use induction on $d=\operatorname{Ndim} M$.

If $d=0, H_{i}^{I}(M, N)=0$ for all $i>p d(M)$ and we have the result.

Let $d>0$. As in the proof of (i) $\Rightarrow$ (ii), there is a positive integer $n$ such that $I^{t} N=I^{n} N$ for all $t \geq n$. Set $K=I^{n} N$, the short exact sequence of artinian $R$-modules

$$
0 \longrightarrow K \longrightarrow N \longrightarrow N / K \longrightarrow 0
$$

induces an exact sequence of generalized local homology modules

$$
\cdots \rightarrow H_{i+1}^{I}(M, N / K) \rightarrow H_{i}^{I}(M, K) \rightarrow H_{i}^{I}(M, N) \rightarrow H_{i}^{I}(M, N / K) \rightarrow \cdots
$$

By the arguments above, $H_{i}^{I}(M, K) \cong H_{i}^{I}(M, N)$ for all $i>p d(M)$. Thus, the proof will be complete if we show that $H_{i}^{I}(M, K)=0$ for all $i>p d(M)+s$. As $I K=K$, there is an element $x \in I$ such that $x K=K$. Now the short exact sequence

$$
0 \longrightarrow 0: K x \longrightarrow K \stackrel{\cdot x}{\longrightarrow} K \longrightarrow 0
$$

induces an exact sequence of generalized local homology modules

$$
\cdots \rightarrow H_{i+1}^{I}(M, K) \rightarrow H_{i}^{I}\left(M, 0:_{K} x\right) \rightarrow H_{i}^{I}(M, K) \stackrel{. x}{\rightarrow} H_{i}^{I}(M, K) \rightarrow \cdots .
$$

As $\operatorname{Ass}_{R}\left(H_{i}^{I}(M, K)\right)=\operatorname{Ass}_{R}\left(H_{i}^{I}(M, N)\right) \subseteq\{\mathfrak{m}\}$ for all $i>p d(M)+s$, the exact sequence yields $\operatorname{Ass}_{R}\left(H_{i}^{I}\left(M, 0:_{K} x\right)\right) \subseteq\{\mathfrak{m}\}$ for all $i>p d(M)+s$. By $[4,4.7], \operatorname{Ndim}\left(0:_{K} x\right) \leq \operatorname{Ndim}\left(0:_{N} x\right) \leq d-1$. Then the inductive hypothesis gives $H_{i}^{I}\left(M, 0:_{K} x\right)=0$ for all $i>p d(\bar{M})+s$ and we have an exact sequence

$$
0 \longrightarrow H_{i}^{I}(M, K) \stackrel{. x}{\longrightarrow} H_{i}^{I}(M, K)
$$

for all $i>p d(M)+s$. If $H_{j}^{I}(M, K) \neq 0$ for some $j>p d(M)+s$, then $\operatorname{Ass}_{R}\left(H_{j}^{I}(M, K)\right)=\{\mathfrak{m}\}$. Thus, there exists a non-zero element $a \in H_{j}^{I}(M, K)$ such that $\mathfrak{m}=\operatorname{Ann}_{R}(a)$, that means $\mathfrak{m} a=0$. Hence $x a=0$, so $a=0$ which is a contraction. Therefore, $H_{i}^{I}(M, N)=H_{i}^{I}(M, K)=0$ for all $i>p d(M)+s$.

(iv) $\Rightarrow(\mathrm{v})$ is clear.

$(\mathrm{v}) \Rightarrow$ (i) by Corollary 2.5 and Lemma 2.6. 


\section{Generalized local cohomology modules}

Note that the $i$-th generalized local cohomology module of $M, N$ with respect to $I$ is defined by Herzog as follows

$$
H_{I}^{i}(M, N)=\underset{t}{\lim } \operatorname{Ext}_{R}^{i}\left(M / I^{t} M, N\right) \text {. }
$$

Let $D(N)=\operatorname{Hom}_{R}(N, E(R / \mathfrak{m}))$ be the Matlis dual of $N$, we have the dual formula.

Lemma 3.1 ([8, 2.3 (ii)]). Let $M$ be a finitely generated module over the local ring $(R, \mathfrak{m})$ and $N$ an $R$-module. Then for all $i \geq 0$,

$$
H_{i}^{I}(M, D(N)) \cong D\left(H_{I}^{i}(M, N)\right) \text {. }
$$

An $R$-module $M$ is called good if its zero submodule has a primary decomposition in $M$. It is clear that finitely generated $R$-modules are good modules.

Theorem 3.2. Let $M$ and $N$ be finitely generated $R$-modules. Let $s$ be a positive integer. Then the following statements are equivalent:

(i) $H_{I}^{i}(M, N)$ is I-stable for all $i<s$;

(ii) $H_{I}^{i}(M, N)$ is finitely generated for all $i<s$;

(iii) $H_{I}^{i}(M, N)$ is good for all $i<s$.

Proof. (i) $\Rightarrow$ (ii). It follows from Lemma 3.1 that $H_{i}^{I}(M, D(N)) \cong D\left(H_{I}^{i}(M, N)\right)$. Then $H_{i}^{I}(M, D(N))$ is $I$-stable for all $i<s$. By Theorem 2.7,

$$
I \subseteq \sqrt{\operatorname{Ann}_{R}\left(H_{i}^{I}(M, D(N))\right.}
$$

for all $i<s$. Then, $I \subseteq \sqrt{\operatorname{Ann}_{R}\left(D\left(H_{I}^{i}(M, N)\right)\right)}=\sqrt{\operatorname{Ann}_{R}\left(H_{I}^{i}(M, N)\right)}$ for all $i<s$. Thus $H_{I}^{i}(M, N)$ is finitely generated for all $i<s$ by $[6,2.9]$.

(ii) $\Rightarrow$ (iii) is clear, since every finitely generated $R$-module is good.

(iii) $\Rightarrow$ (i). We have $H_{i}^{I}(M, D(N)) \cong D\left(H_{I}^{i}(M, N)\right)$. As $H_{I}^{i}(M, N)$ is good for all $i<s, D\left(H_{I}^{i}(M, N)\right)$ is representable for all $i<s$ by [1,3.2]. Thus $H_{i}^{I}(M, D(N))$ is representable for all $i<s$. It follows from Theorem 2.7 that $I \subseteq \sqrt{\operatorname{Ann}_{R}\left(D\left(H_{I}^{i}(M, N)\right)\right)}=\sqrt{\operatorname{Ann}_{R}\left(H_{I}^{i}(M, N)\right)}$ for all $i<s$. Therefore $H_{I}^{i}(M, N)$ is $I$-stable for all $i<s$.

In the following theorem we find some equivalent conditions when generalized local cohomology modules $H_{i}^{I}(M, N)$ are good for all $i>p d(M)+s$.

Theorem 3.3. Let $M$ and $N$ be finitely generated $R$-modules. Let $s$ be a non-negative integer. Then the following statements are equivalent:

(i) $H_{I}^{i}(M, N)=0$ for all $i>p d(M)+s$;

(ii) $H_{I}^{i}(M, N)$ is finitely generated for all $i>p d(M)+s$;

(iii) $\operatorname{Coass}_{R}\left(H_{I}^{i}(M, N)\right) \subseteq\{\mathfrak{m}\}$ for all $i>p d(M)+s$;

(iv) $I \subseteq \sqrt{\operatorname{Ann}_{R}\left(H_{I}^{i}(M, N)\right)}$ for all $i>p d(M)+s$;

(v) $H_{I}^{\bar{i}}(M, N)$ is I-stable for all $i>p d(M)+s$;

(vi) $H_{I}^{i}(M, N)$ is good for all $i>p d(M)+s$. 
Proof. (i) $\Rightarrow$ (ii) is clear.

(ii) $\Rightarrow$ (iii) follows from $[13,2,10]$.

(iii) $\Rightarrow($ iv $)$. We have $H_{i}^{I}(M, D(N)) \cong D\left(H_{I}^{i}(M, N)\right)$. By $[13,1.7]$

$$
\underset{R}{\operatorname{Ass}}\left(D\left(H_{I}^{i}(M, N)\right)=\underset{R}{\operatorname{Coass}}\left(H_{I}^{i}(M, N)\right) \subseteq\{\mathfrak{m}\} .\right.
$$

Then $\operatorname{Ass}_{R}\left(H_{i}^{I}(M, D(N))\right) \subseteq\{\mathfrak{m}\}$. It follows from 3.2 that

for all $i>p d(M)+s$.

$$
\begin{aligned}
I & \subseteq \sqrt{\operatorname{Ann}_{R}\left(H_{i}^{I}(M, D(N))\right)} \\
& =\sqrt{\operatorname{Ann}_{R}\left(D\left(H_{I}^{i}(M, N)\right)\right)} \\
& =\sqrt{\operatorname{Ann}_{R}\left(H_{I}^{i}(M, N)\right)}
\end{aligned}
$$

(iv) $\Rightarrow$ (i). From the hypothesis and the isomorphism $H_{i}^{I}(M, D(N)) \cong$ $D\left(H_{I}^{i}(M, N)\right)$ we get $I \subseteq \sqrt{\operatorname{Ann}_{R}\left(H_{i}^{I}(M, D(N))\right)}$ for all $i>p d(M)+s$. By $\left.2.8, H_{i}^{I}(M, D(N))\right)=0$ for all $i>p d(M)+s$. It follows $D\left(H_{I}^{i}(M, N)\right)=0$ and then $H_{I}^{i}(M, N)=0$ for all $i>p d(M)+s$.

(iv) $\Leftrightarrow(\mathrm{v})$. The arguments are similar to that in the proof of Theorem 3.2.

(i) $\Rightarrow$ (vi) is clear.

(vi) $\Rightarrow(\mathrm{i})$. We have $H_{i}^{I}(M, D(N)) \cong D\left(H_{I}^{i}(M, N)\right)$. Note that Matlis dual of a good $R$-module $R$-module is representable. That means $H_{i}^{I}(M, D(N))$ is representable for all $i>p d(M)+s$. Therefore $H_{i}^{I}(M, D(N))=0$ by Theorem 2.8. That means $D\left(H_{I}^{i}(M, N)\right)=0$ and then $H_{I}^{i}(M, N)=0$ for all $i>$ $p d(M)+s$.

For two $R$-modules $M$ and $N$, we put

$$
\operatorname{ext}^{+}(M, N)=\sup \left\{i \mid \operatorname{Ext}_{R}^{i}(M, N) \neq 0\right\} .
$$

Theorem 3.3 gives us the following consequence.

Corollary 3.4. Let $M$ and $N$ be finitely generated $R$-modules such that $\operatorname{dim} N$ $=d$ and $\operatorname{ext}^{+}\left(M, H_{I}^{d}(N)\right)=p d(M)=r<\infty$. Then $H_{I}^{r+d}(M, N)$ is not good.

Proof. It follows from $[11,2.2]$ that $H_{I}^{r+d}(M, N) \neq 0$ and $H_{I}^{i}(M, N)=0$ for all $i>r+d$. By Theorem 3.3 we have the result.

Acknowledgments. The author would like to express his gratitude to Professor Nguyen Tu Cuong for his support and advice.

\section{References}

[1] H. Ansari-Toroghy, Secondary representation of some modules over a commutative ring, Acta Math. Hungar. 100 (2003), no. 3, 257-262.

[2] M. P. Brodmann and R. Y. Sharp, Local Cohomology: An Algebraic Introduction with Geometric Applications, Cambridge University Press 1998.

[3] N. T. Cuong and T. T. Nam, The I-adic completion and local homology for Artinian modules, Math. Proc. Cambridge Philos. Soc. 131 (2001), no. 1, 61-72. 
[4] - A local homology theory for linearly compact modules, J. Algebra 319 (2008), no. $11,4712-4737$.

[5] J. Herzog, Komplexe, Auflösungen und dualität in der localen Algebra, Habilitationschrift Univ. Regensburg, 1970

[6] K. Khashyarmanesh, M. Yassi, and A. Abbasi, A new version of local-global principle for annihilations of local cohomology modules, Colloq. Math. 100 (2004), no. 2, 213-219.

[7] I. G. Macdonald, Secondary representation of modules over a commutative ring, Symposia Mathematica, Vol. XI (Convegno di Algebra Commutativa, INDAM, Rome, 1971), pp. 23-43. Academic Press, London, 1973.

[8] T. T. Nam, Generalized local homology for artinian modules, Algebra Colloquium (to appear).

[9] Generalized local homology, Proceedings of the 4th Japan-Vietnam Joint Seminar, Meiji University, Japan (2009), 217-235

[10] _ A finiteness result for co-associated and associated primes of generalized local homology and cohomology modules, Comm. Algebra 37 (2009), no. 5, 1748-1757.

[11] _ On the non-vanishing and the artinianness of generalized local cohomology modules, Journal of Algebra and Its Applications (to appear).

[12] S. Rezaei, Some results on local homology and local cohomology modules, Illinois Journal of Mathematics (to appear).

[13] S. Yassemi, Coassociated primes, Comm. Algebra 23 (1995), no. 4, 1473-1498.

Tran TUAN NAM

Ho Chi Minh University of Pedagogy

280 An DuOng Vuong

District 5, Ho Chi Minh City, Vietnam

E-mail address: namtuantran@gmail.com

Nguyen Duc Minh

Industrial University of Ho Chi Minh City

12 NGUYen VAN BaO

Go vap, Ho Chi Minh City, Vietnam

E-mail address: daytoan@gmail.com 